\title{
RIBONUCLEOPROTEIN STRUCTURES IN MEIOTIC PROPHASE OF ZEA MAYS*
}

\author{
MARTA SHERMAN WALTERS \\ Santa Barbara Botanic Garden, Santa Barbara, California, U.S.A., and Department of \\ Biological Sciences, University of California, Santa Barbara
}

Received 15.iii.67

\section{INTRODUGTION}

SEVERAL different structures composed of ribonucleoprotein (RNP) have been observed in the nucleus of Zea mays during meiotic prophase. These ranged from some rather diffuse substance to nebennucleoli of various sizes, and included the structure previously described as a "nuclear body" (Walters, 1965). The various RNP structures appeared at different stages of prophase and diminished and disappeared at the end of prophase. Autoradiographic studies have shown that nuclear RNA synthesis is very active during early meiotic prophase and diminishes in later stages of prophase (Taylor, 1959; Henderson, 1964; Muckenthaler, 1964; Das, 1965). However, these studies were not suited to illustrate changes in cellular morphology that may accompany such alterations in synthetic activity.

The presence of RNP structures in prophase of Zea suggests that RNA is produced faster than it is dispersed. Their appearance in different stages of prophase suggests that these stages differ in rates of formation or utilisation of RNA. However, the presence of several types of structures indicates variation more complex than increase-or-decrease of RNA production. If such structures do not represent different types of synthesis they may at least represent different types of association and release of synthetic products. Moreover, their appearance is difficult to interpret on the basis of the conventional sequence of DNA-RNA-protein.

Some similarities are observed between the RNP structures in Zea and those produced by the actively synthetic polytene chromosomes of dipteran larvae and lampbrush chromosomes of amphibian oocytes (Swift, 1959a; Gall, 1955). In addition, Zea chromosomes at diakinesis have some morphological resemblance to lampbrush chromosomes. The present observations support previous suggestions of lampbrush-like chromosomes in plants (de Castro, 1948; Grun, 1958).

\section{MAterials AND METHODS}

These studies were made with material from two plants of the KYS-strain of Zea mays, 26485-1 and 26489-5. Flower buds were fixed in a mixture of 3 parts 95 per cent. ethyl alcohol:1 part propionic acid and stored in 70 per cent. alcohol under refrigeration. In all instances whole p.m.c.'s were stained. The procedures have been described in previous publications

* This investigation was supported in part by a Public Health Service Research Career Development Award No. K3-GM-729 from the National Institute of General Medical Sciences and in part by Research Grant GB-609 from the National Science Foundation. 
(Walters, 1965, 1966); these included staining with propiono-carmine, Azure B, methyl green-pyronin, as well as tests with alloxan, Feulgen and ribonuclease. In addition, p.m.c.'s were treated with desoxyribonuclease (Worthington, I); experimental slides were placed in DNase, $0.2 \mathrm{mg} . / \mathrm{ml}$. at $p \mathrm{H} 7.0$ and control slides in $\mathrm{MgSO}_{4}$ solution at $p \mathrm{H} 7.0$ for one hour at $37^{\circ} \mathrm{C}$. Test and control slides were hydrolysed in $1 \mathrm{~N} \mathrm{HCl}$ at $60^{\circ} \mathrm{C}$. for 8 minutes and treated with leuco-basic fuchsin as described previously.

\section{Observations}

The presence of RNA in the structures described below was indicated by their metachromatic staining with Azure B and their staining with pyronin in methyl green-pyronin Y. They were not visible in microsporocytes treated with ribonuclease and stained with Azure B. The presence of proteins in these structures was indicated by their staining with leuco-basic fuchsin after treatment with alloxan. They were not stained after the Feulgen test. It has therefore been concluded that the various structures contain ribonucleoprotein and they will be referred to as "RNP structures".

In Zea the attenuated chromosomes occupy the entire nucleus in interphase and leptotene, and form a tight mass on one side of the nucleolus during zygotene and early pachytene. The considerably shortened bivalents emerge from this mass and are well spread at mid-pachytene and later stages of prophase (Rhoades, 1950). There is no prometaphase clump; at the end of diakinesis the bivalents move from their scattered positions to the equatorial plate.

The following descriptions of the RNP structures in meiotic prophase were based primarily on studies of propiono-carmine and Azure B preparations. (All illustrations are from propiono-carmine preparations.)

At leptotene there was a single large nucleolus, and sometimes also small round bodies that were interpreted as nuclear body primordia.

By zygotene a nuclear body was present (Walters, 1965). This body consisted of a number of closely appressed globules with dark-staining walls, often surrounded by a light-staining substance also composed of globules (plate II, b and c); it occurred as a single structure or in two or three parts.

At very early pachytene a few cells contained one small nebennucleolus; this was a small round or oval body that differed from the nuclear body in its homogeneous structure (plate II, a-c). Later, when the chromosomes began to separate from the synizetic knot, a number of nebennucleoli were present (plate I, b). The numbers of nebennucleoli increased from early to mid-pachytene.

A small amount of diffuse material appeared at very early pachytene, and more was present when the chromosomes emerged from the synizetic knot (plate I, a and b). At mid-pachytene the diffuse material graded into more compact bodies and these into nebennucleoli; thus there was an unbroken sequence between diffuse substance and nebennucleoli (plate II, a-d). The quantity of diffuse material increased at late diplotene and during diakinesis. At late diakinesis there were numerous irregular masses, often assembled in clusters or interconnected by strands; these frequently contained darkstaining granules (see below).

At very late diakinesis, when the nucleolus had decreased considerably in size, numerous homogeneous round bodies were present (plate I, c).

From this point on, the RNP structures diminished rapidly. At the end 
of diakinesis the only ones visible were remnants of the nucleolus and nuclear body (plate I, d). In Azure B preparations the metachromatic staining of the nuclear sap increased at late diakinesis as the RNP structures disappeared.

The RNP structures stained with different intensities in Azure B and methyl green-pyronin preparations. The nucleolus was dark-staining and contained lighter circular areas. Many nebennucleoli were dark-staining, including some very small ones. The nuclear body had lighter and darker areas, probably corresponding with those observed after propiono-carmine staining (plate II, b and $c^{c}$ ); in overall appearance it was lighter than the nucleolus and many nebennucleoli until late diakinesis, when the nuclear body and nucleolus appeared equally dark-staining. Much of the free diffuse material at pachytene and irregular masses at diakinesis were lightstaining, but there was an unbroken sequence from light diffuse material to dark nebennucleoli. The large round homogeneous bodies appearing near the end of diakinesis were dark-staining like the nucleolus. The propionocarmine staining of dark and light areas of the nuclear body and diffuse material is shown in plate II, e and $f$, which represent short and long exposures of the same negative. The diffuse material and the light substance surrounding the nuclear body are stained with about the same intensity.

Certain RNP structures were associated with chromosomes, while others were free in the nuclear sap:

Nucleolus. In early prophase the nucleolus was attached to the nucleolar organising region of chromosome 6 (McClintock, 1934).

Nuclear body. The nuclear body often appeared to be associated with a bivalent at mid-pachytene (plate II, b and $c^{c}$ ). However, it was associated with various regions of different bivalents. Furthermore, it often lay at some distance from the chromosome mass at an earlier stage (plate I, a). Therefore associations of the nuclear body with chromosomes at pachytene were probably fortuitous (Walters, 1965).

Nebennucleoli. When nebennucleoli appeared at early pachytene they often lay among or near the chromosomes. But at mid-pachytene nebennucleoli were associated with various bivalents in different microsporocytes. Specific associations of nebennucleoli and chromosomes could not, therefore, be demonstrated.

Diffuse material. At pachytene diffuse material and structures intermediate between diffuse material and nebennucleoli often appeared to be associated with or to emanate from chromosomes (plate II, $\mathrm{a}^{b}, \mathrm{a}^{c}, \mathrm{c}^{a}, \mathrm{~d}^{a}$ and $\left.\mathrm{d}^{b}\right)$. However, some RNP material was free in the nuclear sap before the chromosomes separated from the synizetic knot (plate I, a). Thus any one of these associations may have been fortuitous. Thin diffuse material intimately associated with bivalents (plate II, $\mathrm{a}^{b}$ and $\mathrm{d}^{a}$ ) was not identifiable in Azure B or alloxan preparations, in which microsporocytes could not be well flattened. In propiono-carmine squashes this material stained just like the free diffuse material that was demonstrably composed of RNP.

From late diplotene through most of diakinesis some irregular bodies and masses of diffuse material appeared to be associated with bivalents (plate III, $a-j$, and fig. 1, a-j). Fine strands extending from the chromosomes (see below) often appeared to penetrate into adjacent RNP structures. This may be a real association or one attributable to contiguity of RNP masses and chromosomes with extending strands. 

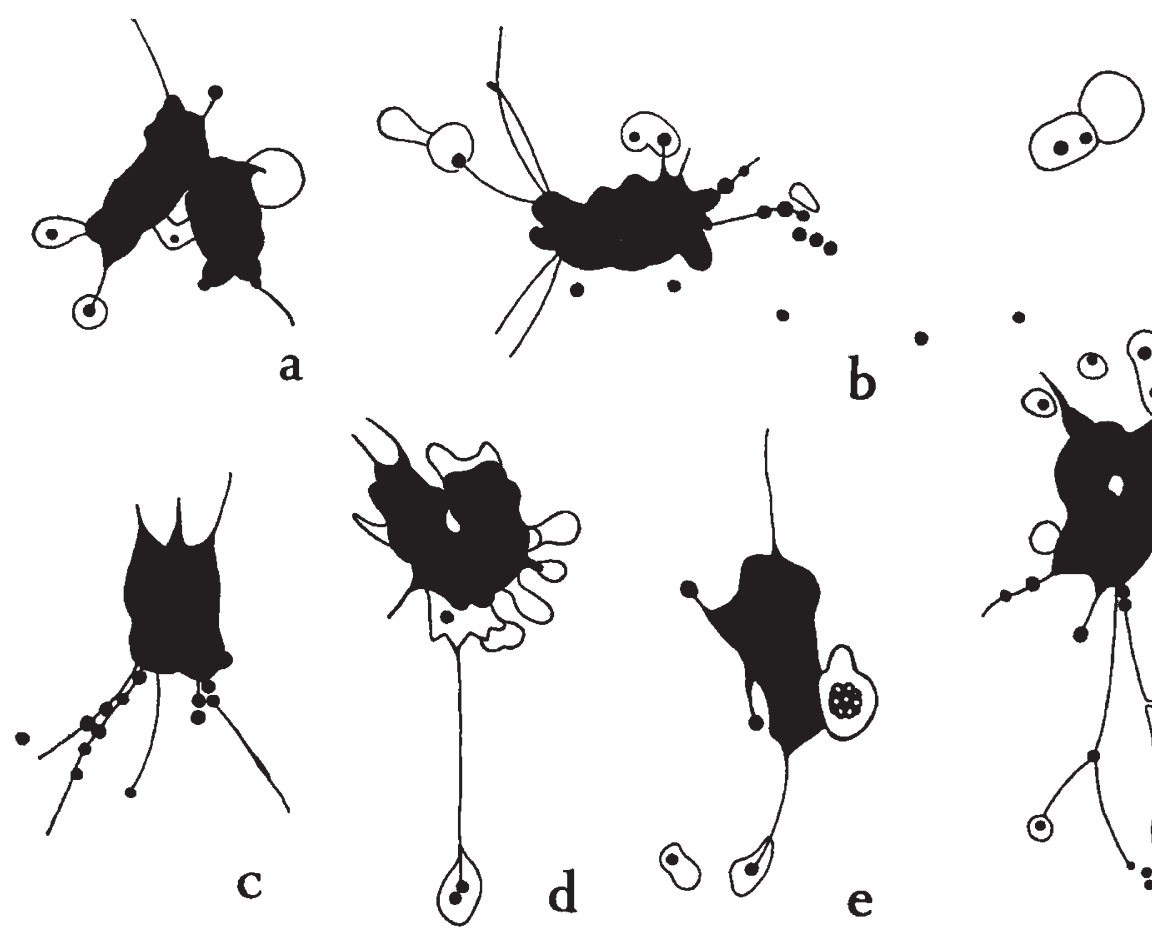

c
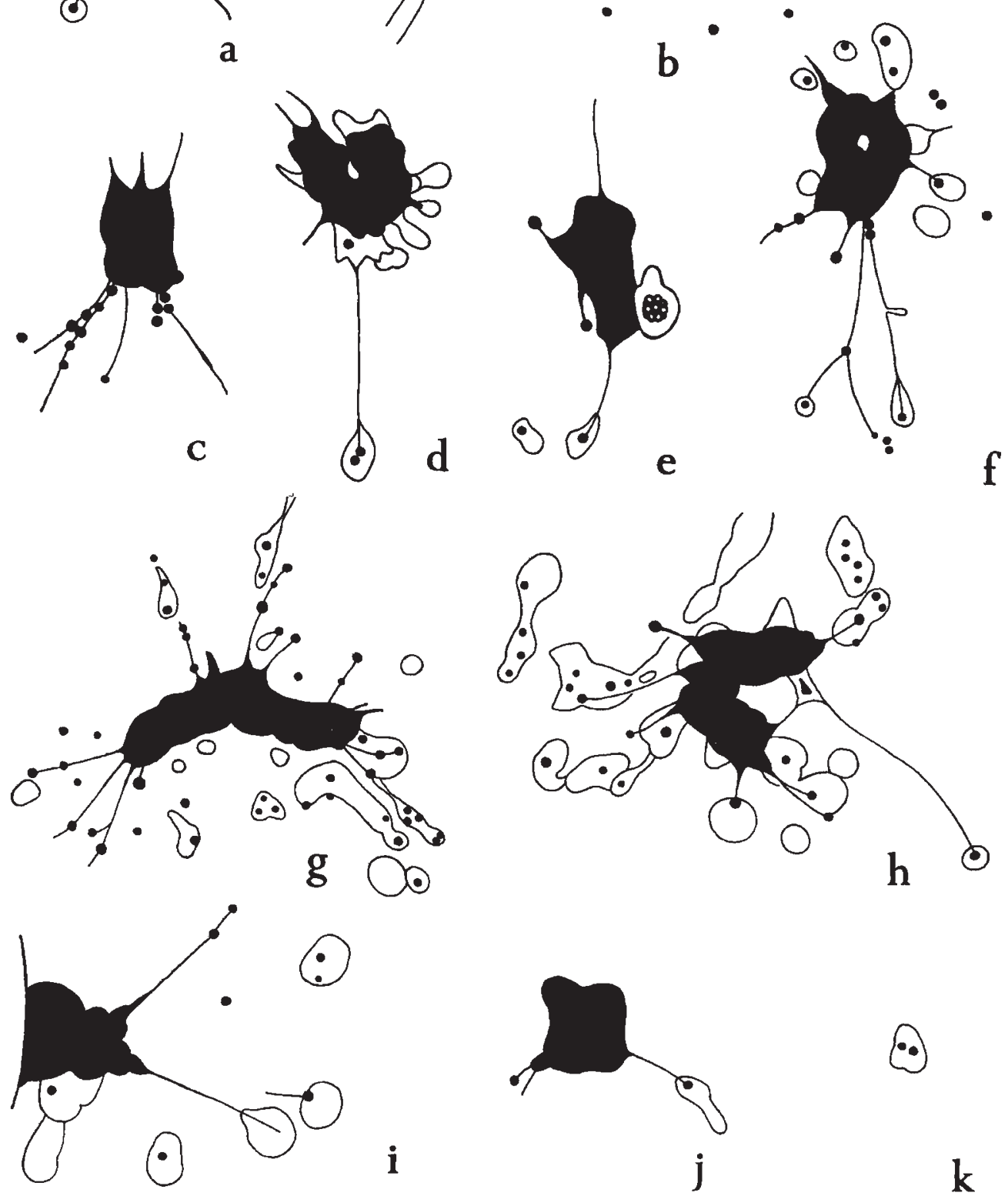
FIG. 1.-(a)-(k) Bivalents and separate granules at diakinesis.
(Corresponding photographs are shown in plate $I I I$, a-k. $)$

$\odot$ 
Near the end of diakinesis there were few apparent associations of chromosome strands with the homogeneous round bodies.

During late diplotene and most of diakinesis the chromosomes had a thready and granular appearance (plate II, h and i; plate III, a-j, and fig. $1, a-j$ ). At late diplotene a few very fine chromosome strands appeared. The strands and granules were difficult to photograph with either brightfield or phase-contrast microscopes. Strands were exceedingly fine; they lay in several focal levels and were lighter-stained after flattening. Some granules and strands were obscured by diffuse material. A number of diakinesis bivalents have therefore been drawn to show strands and granules that are obscure or out of focus in the photographs. From early to late diakinesis numerous strands extended from interstitial and terminal regions of bivalents, so that chromosomes were somewhat "brushy" (plate II, $\mathrm{h}$ and $\mathrm{i}$; plate III, g, and fig. $\mathrm{l}, \mathrm{g}$ ). Strands often appeared to extend from the chromosomes into masses of RNP material (plate III, g and h, and fig. $1, \mathrm{~g}$ and $\mathrm{h}$ ). An occasional granule appeared at late pachytene or diplotene (plate II, f) and these increased in frequency during diakinesis. The granules were approximately $0.3 \mu$ to $0.5 \mu$ in diameter. Some were free and some were contained in the RNP masses described above (plate III, g-k, and fig. 1, g-k). Granules were often associated with chromosome strands, terminally or in a linear arrangement " along " a strand. Strands with numerous granules most often extended from terminal regions of chromosomes (plate III, b and c, and fig. l, b and c). The strands and granules decreased in number during very late diakinesis and disappeared at the end of diakinesis.

The composition of granules and chromosome strands is not known. Strand colour could not be determined in Azure B and methyl greenpyronin preparations. Strands observed after Feulgen treatment may have been coloured or visible by refraction. After exposure to DNase followed by Feulgen treatment, neither chromosomes nor strands were visible. Granules could not be identified in preparations other than propiono-carmine squashes.

The structures described above differed to some extent in the two closely related plants of Zea in which they were observed. There was a greater amount of diffuse material and more numerous nebennucleoli in 26489-5. Chromosome strands, particularly those with associated granules, were more numerous or conspicuous in 26485-1.

\section{Discussion}

Various RNP structures are present in the nucleus during different stages of meiotic prophase of Zea mays. There are two principal sources from which such structures may be derived-the chromosomes and nucleolus. Some structures appear most probably to be derived from the chromosomes, others from the nucleolus, or from combined chromosomal and nucleolar materials.

The chromosomes synthesise RNA during pre-meiotic interphase and meiotic prophase; the rate of synthesis is highest during interphase and early prophase and decreases as chromosomes contract at late prophase (Henderson, 1964; Muckenthaler, 1964; Das, 1965; Das, Siegel and Alfert, 1965). Nucleolar RNA is synthesised by nucleolar organising regions of specific chromosomes (Lin, 1955; Brown and Gurdon, 1964; Ritoss a 
and Spiegelman, 1965). In Zea, nucleolar size and its RNA and protein content increase from leptotene to mid-pachytene and decrease slightly to mid-diplotene and very rapidly throughout diakinesis (Linn, 1955). According to Das (1965) this increase may result from a reduced rate of transport of nucleolar RNA to the cytoplasm. He finds the nucleolar apparatus of Zea synthesises RNA maximally during pre-meiotic interphase; synthesis decreases rapidly in early prophase and ceases in early pachytene before the chromosomes emerge from the synizetic knot.

In Zea the nucleolus forms during pre-meiotic telophase, attached to the organiser region of chromosome 6 (McClintock, 1934). It appears to arise from material synthesised by the organiser. Perhaps the nucleolus may be visualised as a giant "puff" associated with a specific genetic locus, as it is sometimes in polytene cells containing accumulations of RNA of various sizes (Swift, 1959a; Harris, 1965).

The nuclear body appears to form by aggregation of globules arising among the chromosomes at late interphase and leptotene (Walters, 1965, 1966). The origin of primordia during stages of maximal chromosomal RNA synthesis and their distribution among the chromosomes suggests that they arise from the chromosomes. Although nuclear body and chromosome associations appear to be fortuitous at pachytene, individual globules may be associated with specific regions of chromosomes in stages closer to their origin. The nuclear body may thus be comparable with ribonucleoprotein structures synthesised by and associated with polytene chromosomes (Swift, $1959 a)$.

The small homogeneous nebennucleoli appear among the chromosomes during early pachytene. At this stage chromosomes are active in RNA synthesis and nucleolar RNA and protein content have not begun to decrease (Lin, 1955; Das, 1965). The nebennucleoli are reminiscent of the small bodies produced by individual chromosomes after loss or inactivation of the nucleolar organiser (McClintock, 1934; Fischberg and Wallace, 1960), and of certain RNP structures produced by lampbrush and polytene chromosomes (Gall, 1955; Swift, 1959a). It is therefore suggested that the nebennucleoli result from synthetic activities of chromosomes.

Diffuse material is present in the nuclear sap from very early pachytene through most of diakinesis. Diffuse material appearing during pachytene seems more likely to have been released from the chromosomes during their stages of active RNA synthesis than from the nucleolus. However, the masses of RNP present at late diplotene and diakinesis may be derived from the nucleolus, whose RNA content decreases rapidly during these stages (Lin, 1955). Some material may come from the chromosomes, which are still synthetically active at diakinesis (Das, 1965), and whose lampbrush-like appearance (see below) is consistent with synthesis and release of ribonucleoprotein (Callan and Lloyd, 1960; Izawa, Allfrey and Mirsky, 1963). It is suggested that ribonucleoprotein in the form of diffuse material may be released from the chromosomes from early pachytene through late diakinesis, and also from the nucleolus from mid-diplotene to the end of diakinesis, and that diffuse RNP structures in late diplotene and diakinesis may be derived from both sources.

The appearance of round bodies near the end of diakinesis, when the nucleolus is considerably decreased in size, and their nucleolus-like staining suggest that these structures are products of nucleolar breakdown. 
Since variation in RNA and protein synthesis is known to occur during meiotic prophase, the simplest explanation of RNP structures derived from chromosomes depends upon an increase in synthetic activity of the chromosomes. Presumably specific regions of chromosomes are involved in a disproportionate change of activity with the result that the synthetic product is not utilised or dispersed as rapidly as it forms. The variation in amount of excess RNP at different stages of prophase agrees with previous observations that the capacity of p.m.c.'s to perform RNA and protein syntheses is a function of meiotic stage (Hotta and Stern, 1963). However, the appearance of several different RNP structures indicates a more complex situation than that just described and may involve differences in synthesis or different types of association and release of the synthetic products. There is no clear explanation for the existence of the RNP structures described here within the conventional conception of the pathway from DNA to protein. In an evolutionary sense, Harris (1965) has suggested that " a selective mechanism operating on this overproduction might confer biological advantages on the cell ".

Two types of chromosomes that are characterised by high metabolic activity are polytene chromosomes of dipteran larvae and lampbrush chromosomes of amphibian oocytes (Swift, 1959a; Isawa, Allfrey and Mirsky, 1963). These chromosomes produce a variety of RNA structures ranging from granules to nucleoli (Gall, 1955; Swift, 1959a). In Triturus the lampbrush chromosomes have many fine projecting fibres and numerous granules that may be shed into the nuclear sap (Callan, 1963). Granules are also a characteristic feature of meiotic prophase in many Drosophila species with lampbrush-like $\mathrm{Y}$ chromosomes (Beermann, Hess and Meyer, 1963). Granules from $0.2 \mu$ to $0.4 \mu$ in diameter are present in polytene chromosomes of Drosophila and a number of chironomid species and may rarely be free in the nuclear sap (Swift, 1959b; Kalnins, Stich and Bencosme, 1964).

Comparisons have been made of some RNA structures in Zea with those produced by polytene and lampbrush chromosomes. Chromosomes of Zea also have some morphological resemblance to lampbrush chromosomes. Numerous fibres project from terminal and interstitial regions of diakinesis chromosomes and (especially at early to mid-diakinesis) these chromosomes may have a "brushy" appearance. Granules appear to be associated with chromosome fibres and numerous granules are free in the nuclear sap.

Lampbrush-like chromosomes have been described previously in Luzula (de Castro, 1948) and Allium (Grun, 1958), and Grun suggests the phenomenon may be widespread among plants. Lafontaine (1965) proposes that spherical granules observed in interphase and prophase mitotic nuclei of several plant species may be analogous to those produced by lampbrush and polytene chromosomes.

Certain differences are observed in chromosome morphology and in RNP structures in the present two plants of Zea. It is expected that aspects of cellular morphology associated with synthetic activities of chromosomes or the nucleolus will vary among individuals of a population and to some extent among microsporocytes of an anther, in response to differences in development and physiological state of the individuals or microsporocytes. 


\section{SUMmary}

1. Several different structures composed of ribonucleoprotein appear at different stages in nuclei of Zea mays during meiotic prophase. The nuclear body is formed by zygotene; nebennucleoli appear in early pachytene; diffuse material appears from early pachytene through most of diakinesis; homogeneous round bodies arise at very late diakinesis. They all disappear at the end of prophase.

2. It is suggested that the nuclear body and nebennucleoli arise from the chromosomes; diffuse material may arise from the chromosomes from early pachytene through late diakinesis and from the nucleolus from mid-diplotene to the end of diakinesis; round bodies arise from nucleolar breakdown.

3. The appearance of RNP structures is believed to reflect variation in synthesis and dispersal, and perhaps in association and release of synthetic products in different stages of meiotic prophase.

4. The chromosomes of Zea appear to have some morphological resemblance to lampbrush chromosomes and some of the RNP structures in Zea may be comparable with those produced by polytene and lampbrush chromosomes.

Acknowledgments.-I wish to thank Dr Marcus M. Rhoades for the fixed material from which this study was made. I am grateful to Dr Spencer W. Brown, Dr Nirmal K. Das and Dr James L. Walters for critical reading of the manuscript.

\section{REFERENCES}

BeERMANN, W., hess, o., AND meYer, F. 1963. Structure and function of the $Y$ heterochromatin in Drosophila. Proc. XVI Int. Cong. Zool., 4, 283-288.

BROWN, D. D., AND GURDON, J. B. 1964. Absence of ribosomal RNA synthesis in the anucleolate mutant of Xenopus laevis. Proc. Nat. Acad. Sci. (Wash.), 51, 139-146.

Gallan, H. G. 1963. The nature of lampbrush chromosomes. Int. Rev. Cytol., 15, 1-34.

CALLAN, H. G., AND LLOYD, L. 1960. Lampbrush chromosomes of crested newts Triturus cristatus (Laurenti). Roy. Soc. London Phil. Trans. Ser. B, 243, 135-219.

De CASTro, D. 1948. Lampbrush chromosomes in plants. Port. Acta biol., Ser. A, 3, 259-261.

DAS, N. K. 1965. Inactivation of the nucleolar apparatus during meiotic prophase in corn anthers. Exper. Cell Res., 40, 360-364.

DAS, N. K., SIEGEL, ELSIE P., AND ALFERT, M. 1965. Synthetic activities during spermatogenesis in the locust. Four. Cell Biol., 25, 387-395.

FISCHBERG, M., AND WALLACE, H. 1960. A mutation which reduces nucleolar number in Xenopus laevis. In The Cell Nucleus. Butterworths, London.

Gall, J. G. 1955. Problems of structure and function in the amphibian oocyte nucleus. Symp. Soc. Exper. Biol., 9, 358-370.

GRUN, P. 1958. Plant lampbrush chromosomes. Exper. Cell Res., 14, 619-621.

HARRIS, H. 1965. The ribonucleic acids in the nucleus and cytoplasm of animal cells. Endeavour, 24, 50-56.

HENDERSON, s. A. 1964. RNA synthesis during male meiosis and spermiogenesis. Chromosoma, 15, 345-366.

IZAWA, MrTsuo, ALlFrey, v. G., AND MiRSky, A. E. 1963. Composition of the nucleus and chromosomes in the lampbrush stage of the newt oöcyte. Proc. Nat. Acad. Sci. (Wash.), $50,811-817$.

KALNins, V. I., STICH, H. F., AND BENCOSme, s. A. 1964. Fine structure of nucleoli and RNAcontaining chromosome regions of salivary gland chromosomes of chironomids and their interrelationship. Canadian 7. Zool., 42, 1147-1155.

LAFOntaine, J. G. 1965. A light and electron microscope study of small, spherical nuclear bodies in meristematic cells of Allium cepa, Vicia faba, and Raphanus sativus. Four. Cell Biol., 26, 1-17.

LIN, M. 1955. Chromosomal control of nucleolar composition in maize. Chromosoma, 7, 340-370. 
Plate I

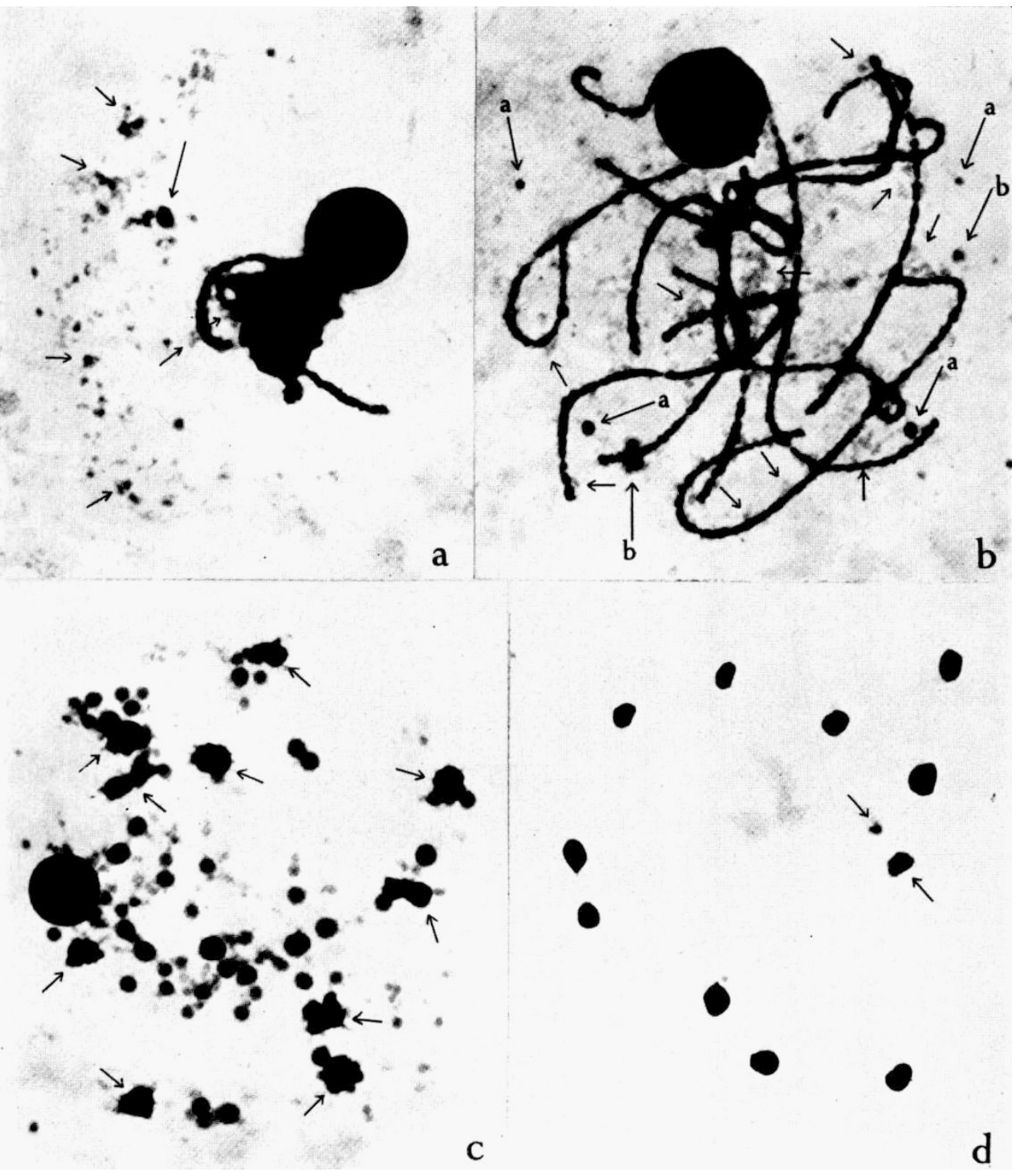

(a) Early pachytene; long arrow indicates nuclear body and short arrows point to diffuse material. $\times 1200$.

(b) Mid-pachytene; arrows $a$ point to nebennucleoli, arrows $b$ to the nuclear body (two parts), and the remaining arrows indicate diffuse material. $\times 1200$.

(c) Very late prophase, showing round bodies; arrows indicate bivalents. $\times 1200$.

(d) End of diakinesis; right arrow indicates remnant of nucleolus and left arrow points to nuclear body. $\times 1200$. 


\section{Plate II}

(a) Pachytene; arrow $a$ indicates nuclear body globule with light-staining material around it; arrows $b$ indicate diffuse material; arrow $c$ points to small nebennucleolus-like structure; arrow $d$ points to nebennucleolus. $\times 3300$.

(b) Pachytene; left arrow indicates small nebennucleolus and right arrow points to nuclear body. $\times 3300$.

(c) Pachytene; arrow $a$ points to small nebennucleolus-like body, arrow $b$ to nebennucleolus and arrow $c$ to nuclear body. $\times 3300$.

(d) Pachytene; arrows indicate different types of diffuse material. $\times 3300$.

(e) Late pachytene, the negative under-exposed to show the structure of the nuclear body, and--

(f) Represents the same negative exposed longer; arrows $a$ indicate dark-staining granules surrounded by light-staining substance, and arrows $b$ point to the nuclear body with light-staining material around the dark-walled centre. $\times 3300$.

(g) Diplotene: left arrow points to nebennucleolus and right arrow to diffuse material. $\times 3300$.

(h), (i) Bivalents at diakinesis. $\times 3300$. 


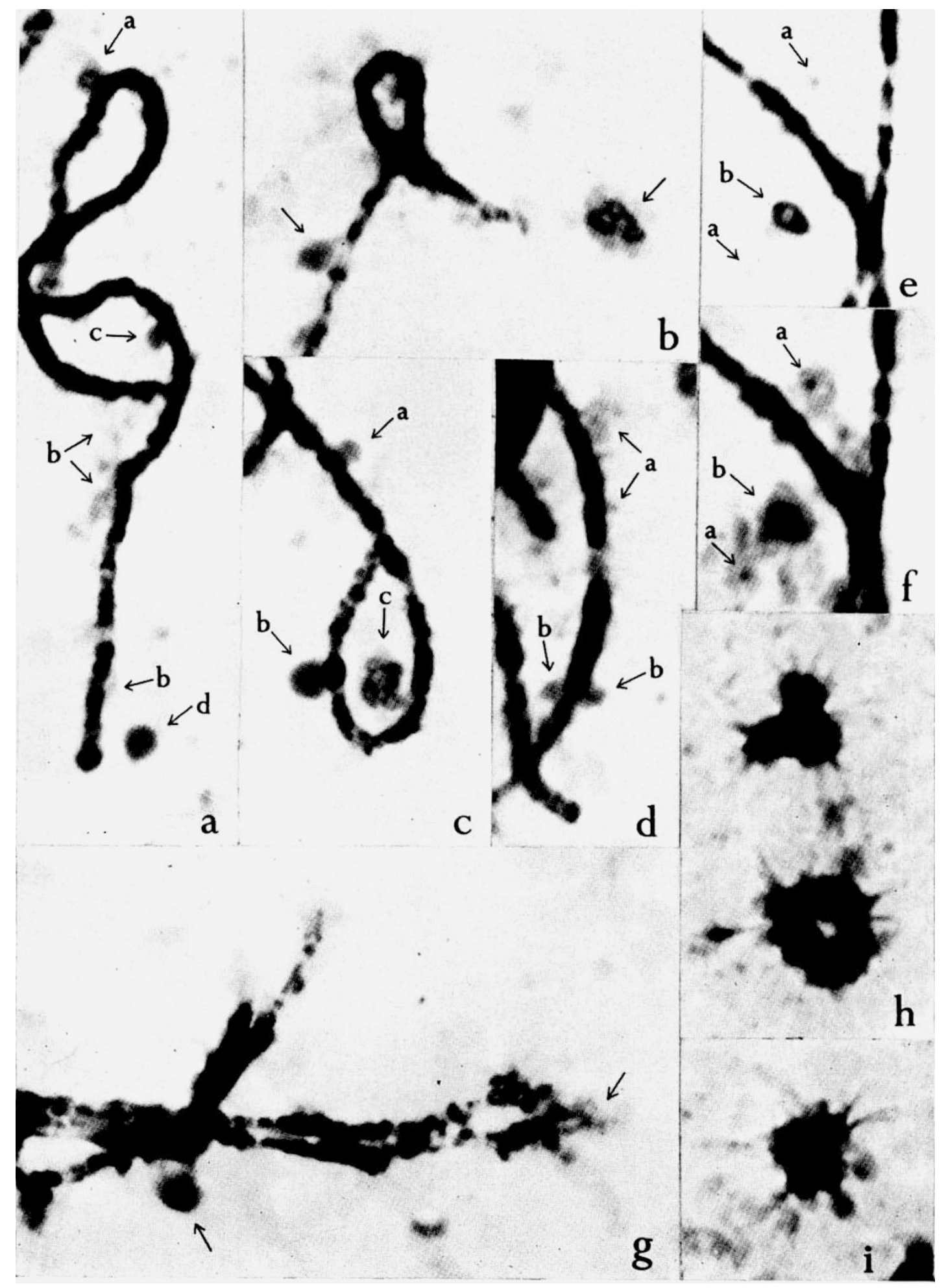


Plate 1 II
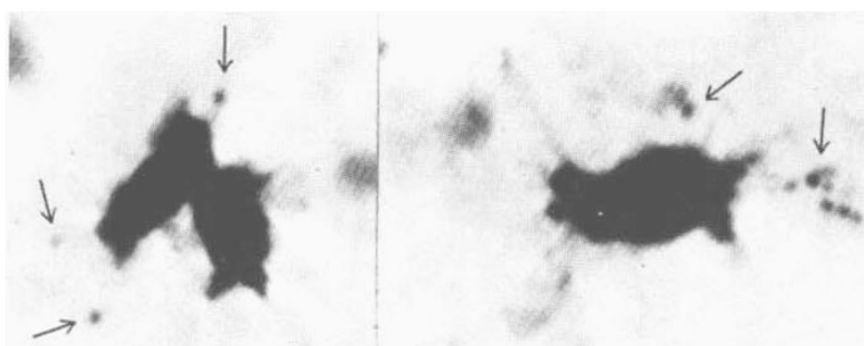

a

$c$

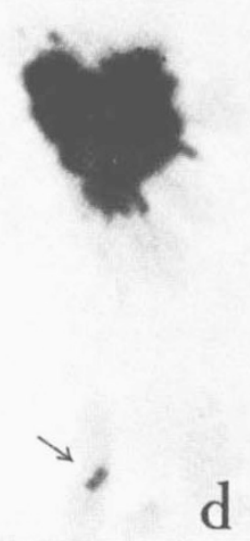

b

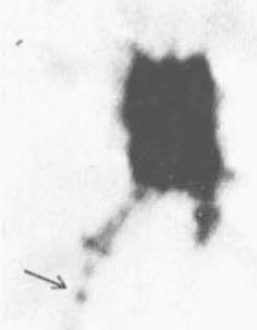

d

$\mathrm{e}$
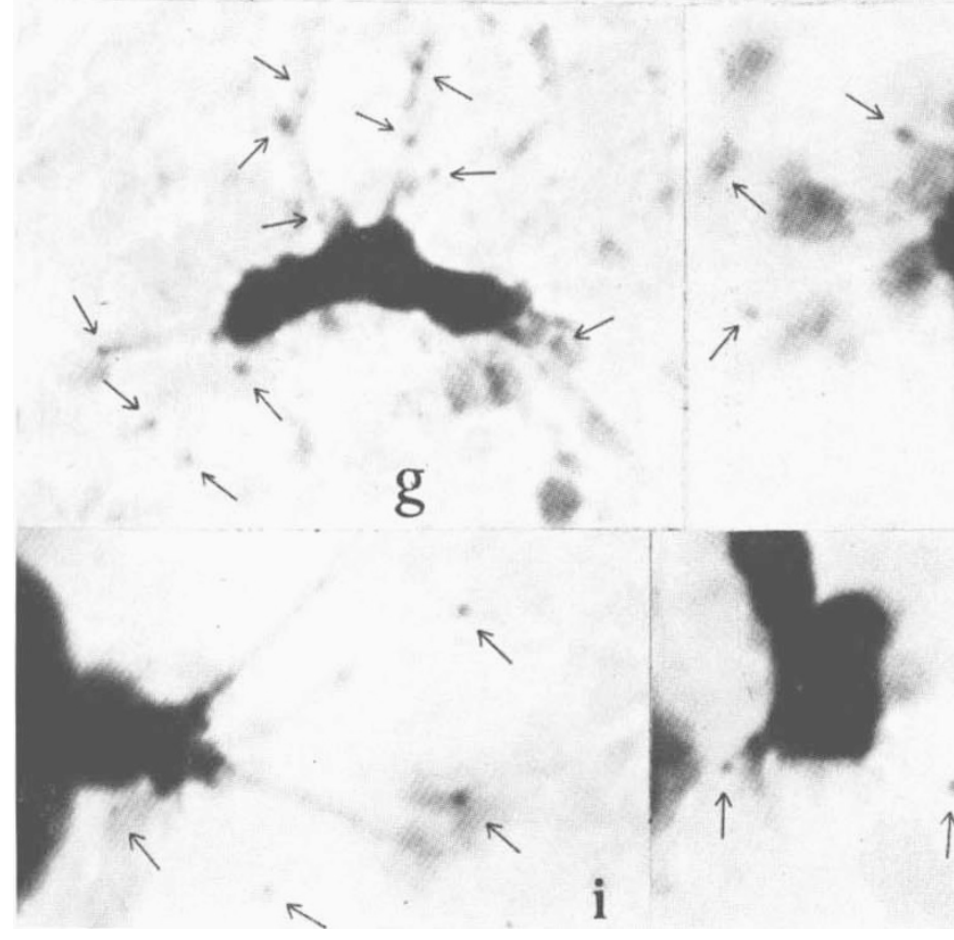

$* \pi$

$\mathrm{h}$
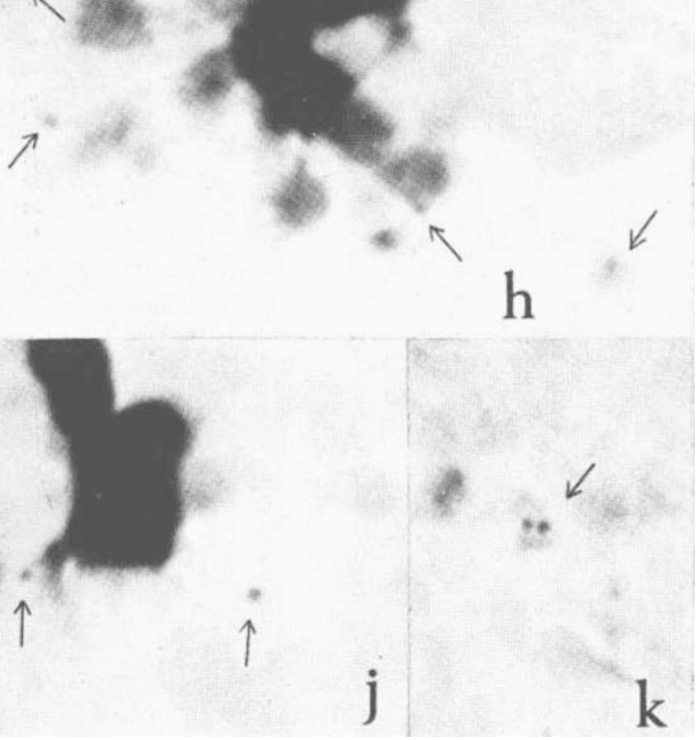

(a) to (k) Bivalents and separate granules at diakinesis. $\times 3300$.

(Corresponding drawings are shown in fig. 1, a-k.) 
MGCLINTOCK, BARBARA. 1934. The relation of a particular chromosomal element to the development of the nucleoli in Zea Mays. Ztschr. Zellf. u. mikr. Anat., 21, 294-328.

MUCKENTHALER, F. A. 1964. Autoradiographic study of nucleic acid synthesis during spermatogenesis in the grasshopper, Melanoplus differentialis. Exper. Cell Res., 35, 531-547. RHOADES, м. м. 1950. Meiosis in maize. 7. Hered., 41, 59-67.

RITOSSA, F. M., AND SPIEGELMAN, s. 1965. Localization of DNA complementary to ribosomal RNA in the nucleolus organizer region of Drosophila melanogaster. Proc. Nat. Acad. Sci. (Wash.) , 53, 737-745.

SWIFT, н. 1959a. Studies on nucleolar function. In A Symposium on Molecular Biology. The University of Chicago Press, Chicago.

SWIFT, H. 1959b. Studies on nuclear fine structure. Brookhaven Symp. Biol., 12, 134-152.

TAYLOR, J. H. 1959. Autoradiographic studies of nucleic acids and proteins during meiosis in Lilium longiflorum. Amer. 7. Bot., 46, 477-484.

WALters, MARTA s. 1965. A nuclear body in microsporocytes of Zea mays. Chromosoma, 17, 78-84.

WALTERS, MARTA s. 1966. Development and chemical constitution of a nuclear body in microsporocytes of Bromus. Heredity, 21, 173-181. 